\title{
Development of Cd-doped Co Nanoparticles En- capsulated in Graphite Shell as Novel Electrode Material for the Capacitive Deionization Tech- nology
}

\author{
Nasser A. M. Barakat ${ }^{1,2, *}$ Khalil Abdelrazek Khalil ${ }^{3}$, Ahmad G. El-Deen ${ }^{4}$, Hak yong kim ${ }^{1, *}$
}

(Received 7 September 2013; accepted 15 October 2013; published online 18 November 2013)

\begin{abstract}
Because of the low energy requirement and the environmentally safe byproducts, the capacitive deionization water desalination technology has attracted the attention of many researchers. The important requirements for electrode materials are good electrical conductivity, high surface area, good chemical stability and high specific capacitance. In this study, metallic nanoparticles that are encapsulated in a graphite shell (Cd-doped $\mathrm{Co} / \mathrm{C} \mathrm{NPs}$ ) are introduced as the new electrode material for the capacitive deionization process because they have higher specific capacitance than the pristine carbonaceous materials. Cd-doped Co/C NPs perform better than graphene and the activated carbon. The introduced nanoparticles were synthesized using a simple sol-gel technique. A typical sol-gel composed of cadmium acetate, cobalt acetate and poly(vinyl alcohol) was prepared based on the polycondensation property of the acetates. The physiochemical characterizations that were used confirmed that the drying, grinding and calcination in an Ar atmosphere of the prepared gel produced the Cd-doped Co nanoparticles, which were encapsulated in a thin graphite layer. Overall, the present study suggests a new method to effectively use the encapsulated bimetallic nanostructures in the capacitive deionization technology.
\end{abstract}

Keywords: Capacitive deionization; Water desalination; Bimetallic nanoparticles; Specific capacitance

Citation: Nasser A. M. Barakat, Khalil Abdelrazek Khalil, Ahmad G. El-Deen and Hak yong kim, "Development of Cd-doped Co Nanoparticles Encapsulated in Graphite Shell as Novel Electrode Material for the Capacitive Deionization Technology", Nano-Micro Lett. 5(4), 303-313 (2013). http://dx.doi.org/10.5101/ nml . v5i4 . p303-313

\section{Introduction}

A shortage of fresh water is one of the largest challenges in the world today. Therefore, desalination technologies must be developed. In the last four decades, the number and capacities of desalination units have dramatically increased such that multi-stage flash (MSF) and reverse osmosis (RO) make up 45\% and $42 \%$, respectively, of world capacity [1]. Neverthe- less, this increase only accounts for one percent of the world's water use, mainly because of the cost [2]. Most existing technologies, such as reverse osmosis and distillation, require a lot of energy. Even nuclear power is too expensive for desalination; in fact, nuclear power is the most expensive water supply option. Among various introduced technologies for water desalination, capacitive deionization (CDI) is a very promising process. CDI (also called electrosorption) attracts enormous atten-

\footnotetext{
${ }^{1}$ Organic Materials and Fiber Engineering Department, Chonbuk National University, Jeonju 561-756, Korea

${ }^{2}$ Chemical Engineering Department, Minia University, El-Minia, Egypt

${ }^{3}$ Mechanical Engineering Department, (NPST), King Saud University, P.O. Box 800, Riyadh 11421, Saudi Arabia

${ }^{4}$ Dept of Bionano System Engineering, College of Engineering, Chonbuk National University, Jeonju 561-756, Korea

*Corresponding authors. E-mail: nasser@jbnu.ac.kr, khy@jbnu.ac.kr
} 
tion as an energy-saving and environmentally friendly desalination technique: it can be conducted at ambient conditions and low voltage without secondary waste, and it does not require high-pressure pumps, membranes, desalination columns or thermal heaters [3-6]. CDI is a technology that is used to remove the dissolved salts from water using the electrosorption of ions onto oppositely charged electrodes. Typically, a brackish water stream flows between pairs of high-surface-area electrodes, which are held at a small potential difference $(\sim 1.3 \mathrm{~V})$. Cations and anions are drawn toward the cathode and the anode, respectively [7].

A high-capacitive electrode should have a high surface area to accumulate ions, a good electrical conductivity to effectively hold charges, a suitable pore size and a good cross-linked structure for smooth ionic motions and electrolyte wetting [8,9]. Carbon materials such as activated carbon (AC) and its composites [10,11], activated carbon cloth (ACC), carbon aerogel $[12,13]$, carbon nanotubes (CNT) and its carbon nanofiber (CNF) composites [14], and ordered and mesoporous carbon (OMC) $[8,15]$ have been studied as electrode materials for CDI. Recently, because of their distinct characteristics, graphene [16] and its composites [3,17-19] have been used by some researchers as effective electrodes for CDI and have yielded better results than other carbonaceous materials.

In addition to the aforementioned required characteristics of CDI electrode materials, the capacitance also has a strong effect; thus, researchers began synthesizing carbon-based composites to improve the specific capacitance, and this new class of materials shows good performance. For example, $\mathrm{MnO}_{2}$ distinctly improves the performance of some carbonaceous materials $[17,20]$. Other materials have also been used to enhance the capacitance of different carbonaceous materials such as $\mathrm{ZnO}[21]$ and $\mathrm{TiO} 2[22,23]$.

Pristine metal nanoparticles (NPs) are expected to have higher electrical conductivity than metal oxides. However, there are two main dilemmas with using pristine metallic NPs in the CDI units: the low chemical stability in water for most conventional metals and the low semiconducting characteristic of the pristine metallic NPs, which leads to a small capacitance. Poly(vinyl alcohol) (PVA) is a semi-crystalline compound with a relatively high carbon content (approximately 54.5\%), and it easily splits the hydroxyl groups in the polymer chain, which makes PVA favorable as a precursor to produce carbonaceous materials. However, the low yield is the main constraint. The decomposition of PVA at temperatures slightly higher than its melting point causes the low carbonization yield. Recently, it was reported that cobalt distinctly enhances the graphitization of PVA and produces metallic nanostructures that are embedded in a graphite shell $[24,25]$. The formed graphite shell strongly enhances the chemical stability, which overcomes the first dilemma. In addition, Cd-doped Co NPs have high specific capacitance [26]. Therefore, the Cd-Co-C system can be a suitable candidate for the CDI process

The introduced catalyst in this study was synthesized using a simple sol-gel technique with poly(vinyl alcohol), cobalt acetate and cadmium acetate as the precursors. To properly evaluate the introduced material, the results were compared to those of activated carbon and graphene, which was prepared using the chemical route [27]. The introduced NPs showed higher desalination performance than graphene and activated carbon.

\section{Experimental}

\section{Materials}

To prepare Cd-doped Co/C NPs, cobalt (II) acetate tetra-hydrate (CoAc, $98 \%$ assay Junsei Chemical Co., Ltd, Japan), cadmium acetate dihydrate (CdAc, 99.0\% assay, Sigma Aldrich), and poly(vinyl alcohol) $(\mathrm{PVA}$, molecular weight $=65000 \mathrm{~g} / \mathrm{mol}$, DC Chemical Co., Ltd, South Korea) were used without modification. Distilled water was used as a solvent. However, graphene was synthesized using graphite powder $(<20 \mu \mathrm{m})$, hydrogen peroxide, hydrazine monohydrate and sulfuric acid (95-97\%), which were purchased from Sigma-Aldrich. N,N-dimethylformamide (DMF 99.5\% assay; SAMCHUN Pure Chemical Co., South Korea) was used as a solvent without modification.

\section{Procedures}

\section{Cd-doped Co/C nanoparticles}

To prepare the CdAc/CoAc/PVA sol-gel, $22.5 \mathrm{~g}$ of PVA (10 wt\%), $5 \mathrm{~g}$ of CoAc (20 wt\%) and $2.5 \mathrm{~g}$ of $\mathrm{CdAc}(20 \mathrm{wt} \%)$ aqueous solutions were well mixed; the final colloid was stirred at $50^{\circ} \mathrm{C}$ for $5 \mathrm{~h}$. The well mixed gel was dried in two successive steps. First, it was dried under atmospheric pressure at $60^{\circ} \mathrm{C}$ for $24 \mathrm{~h}$, then under high vacuum atmosphere at $80^{\circ} \mathrm{C}$ for $48 \mathrm{~h}$. The obtained solid material was crushed and grinded. The calcination process was performed under argon atmosphere at $1 \mathrm{~atm}$ and $700^{\circ} \mathrm{C}$ for $5 \mathrm{~h}$ with a heating rate of $2.3^{\circ} \mathrm{C} / \mathrm{min}$.

\section{Preparation of graphene oxide (GO)}

Graphene was chemically prepared by reducing exfoliated graphene oxide (GO). The GO was synthesized from natural graphite powder using a modified Hummer's method $[28,29]$. A brief description of the procedure: $5 \mathrm{~g}$ of graphite, which were treated twice with $5 \% \mathrm{HCl}$, were placed in cold $\left(0^{\circ} \mathrm{C}\right)$ concentrated $\mathrm{H}_{2} \mathrm{SO}_{4}$ $(130 \mathrm{~mL}) ; 15 \mathrm{~g}$ of $\mathrm{KMnO}_{4}$ were gradually added to the mixture in an ice bath and stirred for $2 \mathrm{~h}$; after the dilu- 
tion with the DI water, the temperature was increased to $98^{\circ} \mathrm{C}$; the mixture was cold to room temperature; then, $\mathrm{H}_{2} \mathrm{O}_{2}(50 \mathrm{~mL}, 30 \mathrm{wt} \%)$ was added, and the mixture was left overnight. The mixture was filtered under vacuum and washed with $10 \%$ aqueous $\mathrm{HCl}$ several times. Finally, it was dried at $50^{\circ} \mathrm{C}$.

\section{Reduction of the exfoliated GO}

In a $250 \mathrm{~mL}$ round flask, $400 \mathrm{mg}$ of the prepared GO were dispersed in $200 \mathrm{~mL}$ distilled water and ultrasonicated for $30 \mathrm{~min}$; then, $0.5 \mathrm{~mL}$ of hydrazine hydrate were added to the suspended GO and microwaved at $1100 \mathrm{~W}$ for $6 \mathrm{~min}$. The bright yellow solution changed to black, which indicated that the reduction process occurred. The precipitated graphene sheets were filtered and washed with distilled water many times; then, they were drayed at $50^{\circ} \mathrm{C}$ for $24 \mathrm{~h}$.

\section{Characterization}

The information about the phase and the crystallinity was obtained using a Rigaku X-ray diffractometer (XRD, Rigaku, Japan) with $\mathrm{Cu} \mathrm{K} \alpha(\lambda=1.5406$ $\AA)$ radiation over the Bragg angle range of $10-100^{\circ}$. Normal- and high-resolution (HR) images were obtained using a transmission electron microscope (TEM, JEOL JEM-2010, Japan), which was operated at 200 $\mathrm{kV}$ and equipped for Energy dispersive X-ray spectroscopy (EDS) analysis. The surface composition was detected using X-ray photoelectron spectroscopy analysis (XPS, AXIS-NOVA, Kratos Analytical, UK) with the following conditions: the base pressure was $6.5 \times 10^{-9}$ Torr, the resolution (pass energy) was $20 \mathrm{eV}$, and the scan step was $0.05 \mathrm{eV} /$ step. The Raman spectra were measured using a Nanofinder 30 spectrometer (Tokyo Inst. Co., Japan), which was equipped with a He:Ne (lambda $=633 \mathrm{~nm}$ ) laser, and the scattering peaks were calibrated with a reference peak from an $\mathrm{Si}$ wafer $\left(520 \mathrm{~cm}^{-1}\right)$. The Raman spectra were recorded under a microscope with a 40x objective in the range of $0-1600 \mathrm{~cm}-1$ and a power of $3 \mathrm{~mW}$ at the sample. The thermal properties were studied using a thermal gravimetric analyzer (TGA, Pyris1, PerkinElmer Inc., USA). The electrochemical measurements were performed on a VersaSTAT 4 (USA) electrochemical analyzer and a conventional three-electrode electrochemical cell. A Pt wire and an $\mathrm{Ag} / \mathrm{AgCl}$ electrode were used as the auxiliary and the reference electrodes, respectively. All potentials were quoted regarding to the $\mathrm{Ag} / \mathrm{AgCl}$ electrode. A glassy carbon electrode was used as the working electrode. The working electrode was prepared by mixing $2 \mathrm{mg}$ of the functional material, $20 \mu \mathrm{L}$ of the Nafion solution ( $5 \mathrm{wt} \%$ ) and $400 \mu \mathrm{L}$ of isopropanol. The slurry was sonicated for $30 \mathrm{~min}$ at room temperature. Fifteen microliters of the prepared slurry was poured onto the active area of the glassy carbon elec- trode, which was then dried at $80^{\circ} \mathrm{C}$ for $20 \mathrm{~min}$.

\section{Results and discussion}

\section{Characterization of Cd-doped $\mathrm{Co} / \mathrm{C}$ nanoparti- cles}

Some metal salts such as chloride, nitrate and acetates can be hydrolyzed and polycondensed to form the gel networks. Using these salts, a viscous polymer can enhance the formation of the gel morphology. In this study, acetate salts were used (typically cobalt and cadmium acetates), and PVA was used as a polymeric matrix. The polycondensation reaction can be explained as follows [30].

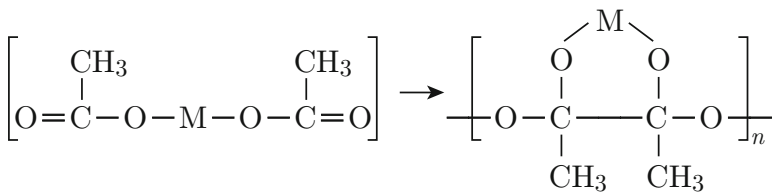

where $\mathrm{M}$ is $\mathrm{Co}$ or $\mathrm{Cd}$. The used precursors formed a good gel, which was dried, ground and calcined as described in the experimental section. The achieved polycondensation operation helps obtaining a highly homogeneous solution and a good distribution of the used metal precursors. The drying of the prepared solgel evaporated the water and yielded a solid material, which could be ground in several steps.

The typical XRD pattern of the calcined powder is shown in Fig. 1, where the standard cobalt peaks are observed. The strong diffraction peaks at the $2 \theta$ values of $44.35^{\circ}, 51.65^{\circ}, 75.95^{\circ}, 92.35^{\circ}$ and $97.75^{\circ}$, which correspond to the (111), (200), (220) (311) and (222) crystal planes, indicate the formation of cubic crystalline cobalt (JCDPS, card no 15-0806). In addition, the broad peak at the $2 \theta$ value of $26.3^{\circ}$ corresponds to an experimental $d$ spacing of $3.37 \AA$, which indicates the presence of graphite-like carbon ( $d$ (002), JCPDS; 41-1487). The XRD spectra also show that no cobalt oxide or carbide phases were present. The peaks that are related to cadmium compounds might be absent

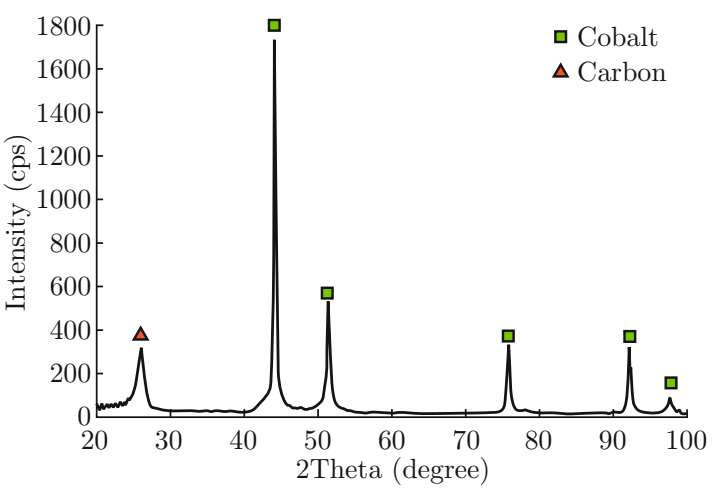

Fig. 1 XRD results for the prepared Cd-doped Co/C NPs. 
because the produced cadmium-based nanoparticles (NPs) were small.

The TGA in the argon atmosphere was performed to explain the formation mechanism of pure cobalt from cobalt acetate/PVA composite. The formation of pristine metal upon heating some metal acetate in an inert atmosphere was previously reported [25,33]. The corresponding phase changes could be supported by thermo-gravimetric studies. The phase changes were examined using the peaks in the first derivative of the TGA curve. Figure 2 shows the TGA results for the $\mathrm{CdAc} / \mathrm{CoAc} / \mathrm{PVA}$ powder in the argon atmosphere. To precisely study these steps, the first derivative data were determined and demonstrated in the same figure. As shown in the first derivative data, there are many apparent peaks in the curve; the first and the second peaks (at $\sim 70^{\circ} \mathrm{C}$ and $\sim 175^{\circ} \mathrm{C}$, respectively) can be attributed to the liberation of physical (moisture) and chemical (combined water molecules with $\mathrm{CoAc}$ and $\mathrm{CdAc}$ ) water from the sample, respectively [25,34]. In fact, the decompositions of CoAc and CdAc are not similar. For example, CdAc decomposes directly to metallic cadmium as follows [34],

$$
\begin{aligned}
& \mathrm{Cd}\left(\mathrm{CH}_{3} \mathrm{COO}\right)_{2} \cdot 2 \mathrm{H}_{2} \mathrm{O} \rightarrow \mathrm{Cd}\left(\mathrm{CH}_{3} \mathrm{COO}\right)_{2}+2 \mathrm{H}_{2} \mathrm{O} \\
& \mathrm{Cd}\left(\mathrm{CH}_{3} \mathrm{COO}\right)_{2} \rightarrow \mathrm{Cd}+0.5 \mathrm{O}_{2}+\mathrm{CH}_{3} \mathrm{COCH}_{3}+\mathrm{CO}_{2}
\end{aligned}
$$

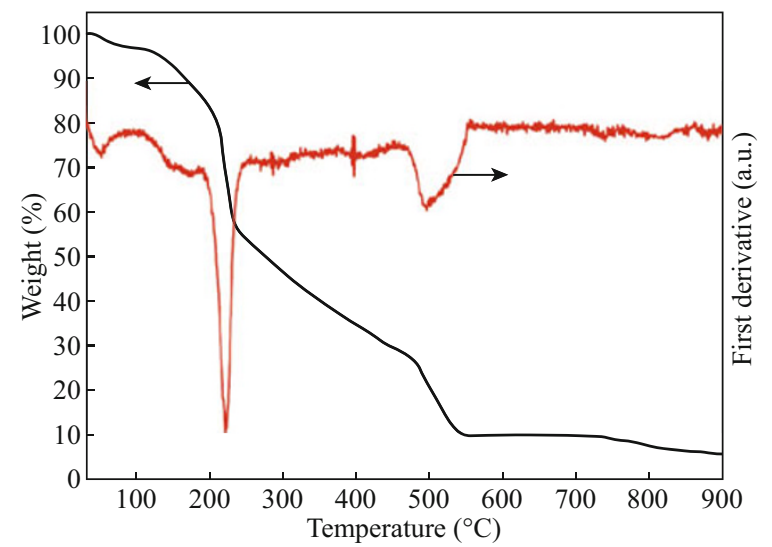

Fig. 2 TGA along with the first derivative for the CdAc/ CoAc/PVA sol-gel.

The metallic cadmium that was formed during the decomposition is notably active and instantaneously reacts with the oxygen that was formed in the same reaction or with traces of oxygen in the inert atmosphere to form $\mathrm{CdO}$. Moreover, it is expected that the remaining cadmium metal will evaporate during the calcination process, which was performed at $700^{\circ} \mathrm{C}$, because it has a low melting temperature $\left(320^{\circ} \mathrm{C}\right)$. Therefore, the cadmium acetate is believed to fully decompose among the first three peaks in the first derivative curve (Fig. 2). However, CoAc decomposes in successive steps. First, the basic cobalt acetate is formed [35].

$$
\begin{aligned}
& \mathrm{Co}\left(\mathrm{CH}_{3} \mathrm{COO}\right)_{2} \cdot 4 \mathrm{H}_{2} \mathrm{O} \rightarrow \\
& \mathrm{Co}(\mathrm{OH})\left(\mathrm{CH}_{3} \mathrm{COO}\right)+3 \mathrm{H}_{2} \mathrm{O}+\mathrm{CH}_{3} \mathrm{COOH}
\end{aligned}
$$

Because the obtained cobalt basic acetate is relatively more stable, it needs more heat to decompose. The third peak (at $\sim 225^{\circ} \mathrm{C}$ ) can be attributed to the decomposition stage of this compound according to the following reaction:

$$
\begin{aligned}
& \mathrm{Co}(\mathrm{OH})\left(\mathrm{CH}_{3} \mathrm{COO}\right) \rightarrow \\
& 0.5 \mathrm{CoO}+0.5 \mathrm{CoCO}_{3}+0.5 \mathrm{H}_{2} \mathrm{O}+0.5 \mathrm{CH}_{3} \mathrm{COCH}_{3}
\end{aligned}
$$

Because the maximum weight loss is obtained at approximately $225^{\circ} \mathrm{C}$, according to the thermal decomposition data of pristine PVA [25], it is acceptable to say that this peak also represents the destruction of this polymer. The broad peak (at $\sim 510^{\circ} \mathrm{C}$ ) can be attributed to the decomposition of the synthesized cobalt carbonate into cobalt monoxide (Eq. (6)). Note that the resulting acetic acid in Eq. (3) decomposes into some gases including reducing gases $\left(\mathrm{CO}\right.$ and $\left.\mathrm{H}_{2}\right)$ [35]. Thus, the formed cobalt oxide is reduced to cobalt metal. Accordingly, the following reactions occurred at this broad peak

$$
\begin{aligned}
& \mathrm{CoCO}_{3} \rightarrow \mathrm{CoO}+\mathrm{CO}_{2} \\
& \mathrm{CoO}+\mathrm{CO} \rightarrow \mathrm{Co}+\mathrm{CO}_{2}
\end{aligned}
$$

Then, a low-rate reduction reaction occurred, which explains the continuous decrease in weight after the last peak. This result implies that the reduction of cobalt monoxide occurs at a small rate in a wide temperature range. Because cobalt is an active transition metal, we cannot expect the formed cobalt monoxide to thermally self-decompose at the used temperature without the aid of a reducing gas (or gases); this conclusion is consistent with reaction $(7)$.

XPS is a unique elemental analysis technique that can provide the chemical-state information of the detected elements, such as the difference between the sulfate and sulfide forms of sulfur. The process works by irradiating a sample with monochromatic X-rays, which results in the emission of photoelectrons whose energies are characteristic of the elements in the sampling volume. Figure 3(a) shows the XPS analysis survey of the obtained powder. The survey scans were performed to search for particular elements in the sample. The survey scan indicated the presence of Co $3 \mathrm{P} 3 / 2(\sim 59 \mathrm{eV})$ [36], C $1 S(\sim 285 \mathrm{eV})$ [37], O $1 s$ and Cd $3 d$ core levels. Figure 3(b), 3(c) and 3(d) show the small-scan-rate investigations at the range of $\mathrm{Cd} 3 \mathrm{~d}_{3 / 2}, \mathrm{C} 1 S$ and $\mathrm{O} 1 S$ orbits, respectively. According to the XPS data, one can claim that the cadmium in the introduced powder is in the form of $\mathrm{CdO}$, which is consistent with the TGA data analysis. The inset shows that the Cd $3 d$ region (Fig. 3(b)) consists of the main $3 d_{3 / 2}$ spin-orbit components at the binding energies of 402 and $409 \mathrm{eV}$, respectively $[38,39]$. Moreover, the O $1 S$ peak appeared as shown in Fig. 3(d), which confirms the formation of $\mathrm{CdO}$. Considering that XPS is a surface analysis technique, from the peak intensities of $\mathrm{Co}$ and $\mathrm{Cd}$, one can 

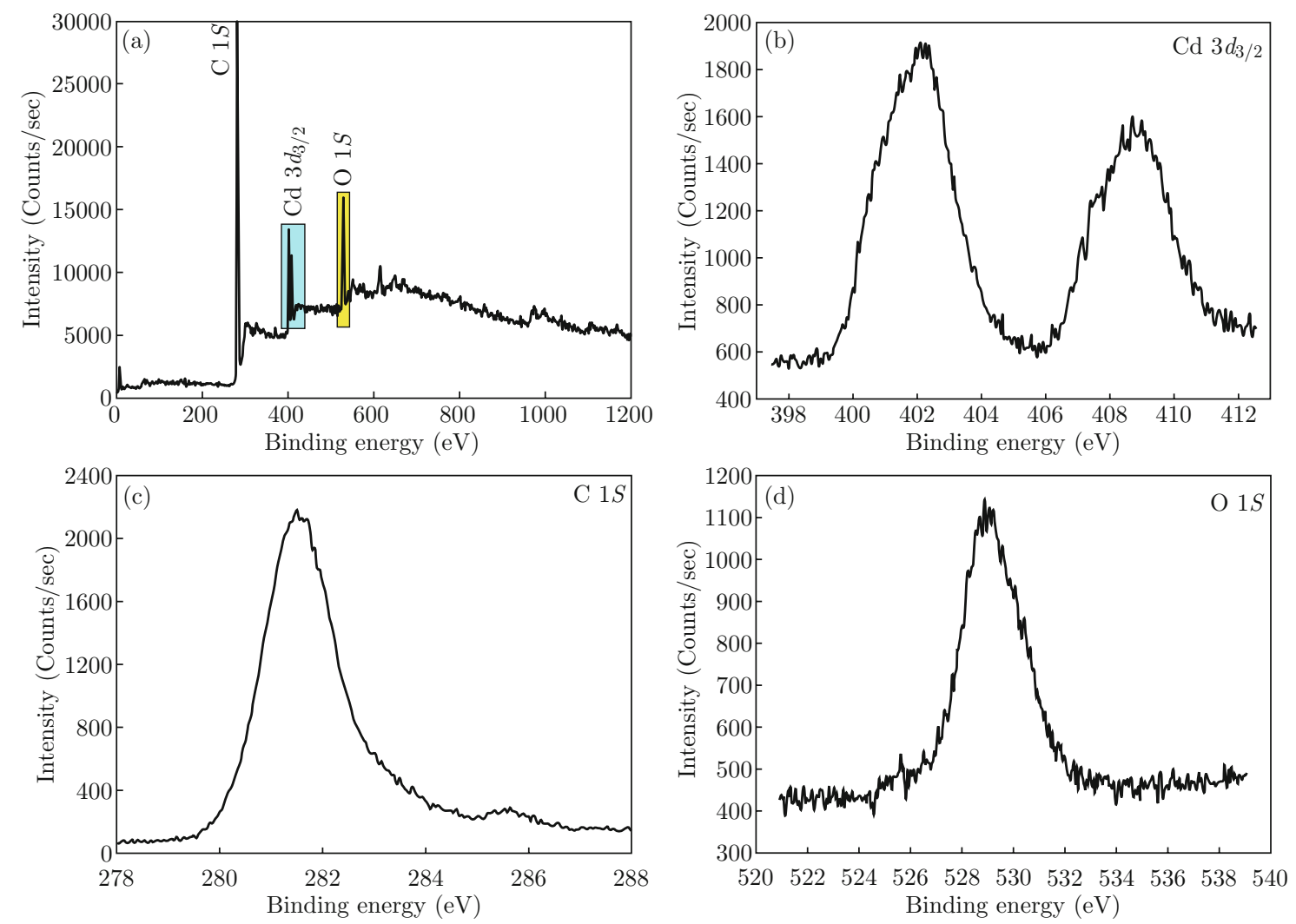

Fig. 3 XPS results for the prepared Cd-doped Co/C NPs. (a) The whole survey; (b), (c) and (d) are the low scan rate investigations for $\mathrm{Cd} 3 d_{3 / 2}, \mathrm{C} 1 S$ and $\mathrm{O} 1 S$ orbits, respectively.
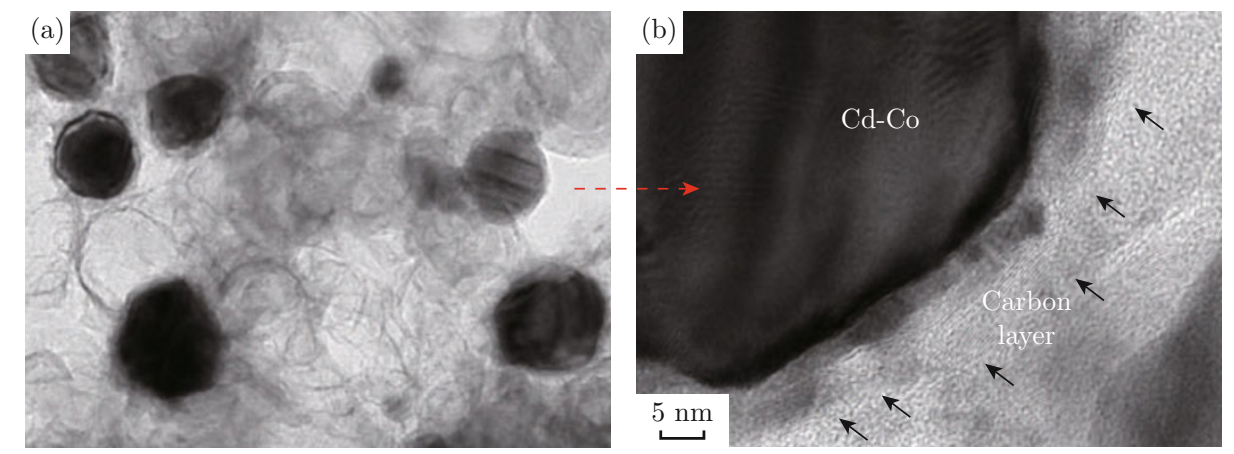

Fig. 4 TEM (a) and HRTEM (b) images for the prepared Cd-doped Co/C NPs. The arrows clarify the carbon layer enveloping.

claim that there is relatively more cadmium than cobalt on the surface of the introduced nanoparticles. Therefore, it can be concluded that the formed $\mathrm{CdO}$ in the surface layer came from the oxidation of $\mathrm{Cd}$ vapor as mentioned in the TGA data discussion. Figure 4 displays the TEM images of the obtained powder. As shown in the figures, metallic nanoparticles that are enclosed in a graphite shell are obtained. Note that the distance between the graphite layers was determined to be $0.32 \mathrm{~nm}$, which almost matches the standard value of $0.335 \mathrm{~nm}$ [40]. The average thickness of the graphite shell is approximately $4.5 \mathrm{~nm}$. Moreover, from Fig. 4(b), one can claim that the metallic nanoparticles are sheathed inside a good-crystallinity thin graphite layer. The envelopment of the cobalt nanostructures in the graphite shell was introduced by the same authors $[25]$.

A good electric conductivity is an important parameter for electrocatalytic materials. Current-voltage plots can provide significant information regarding a material's conduction mechanisms and breakdown strengths. Figure 5 shows the $I-V$ plots for a thin film from the introduced material on the silicon substrate. The $I$ $V$ plot clearly resembles the metallic conductors. This finding indicates the high conductivity of the formed graphite shells and confirms the formation of metallic 


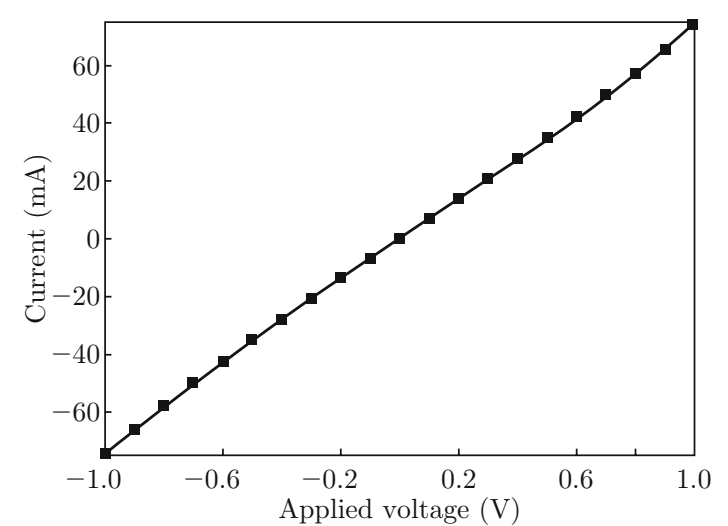

Fig. 5 The electrical conductivity measurement for the introduced nanoparticles.

NPs as the main constituent of these materials.

\section{Reduced graphene oxide characterization}

Graphene is an intriguing 2D monolayer carbon sheet whose distinct properties make it notably promising in various applications. Since the first report of graphene production using a mechanical exfoliation method, many techniques that are broadly classified as top-down and bottom-up syntheses have been developed. Among the various reported methods to produce graphene, the chemical reduction of graphite oxide (GO) or chemically converted graphenes is an established, low-cost, scalable and highly flexible method to chemically functionalize graphene materials $[41,42]$. Therefore, in this study, the chemical route was selected. An important property of GO, which is introduced by the hydrophilic nature of oxygenated graphene layers, is its easy exfoliation in aqueous media. As a result, GO readily forms stable colloidal suspensions of thin sheets in water [43]. After a suitable ultrasonic treatment, such exfoliation can produce stable dispersions of notably thin graphene oxide sheets in water [41]. Therefore, the colloidal suspension of the GO that was prepared with ultrasound was clear, homogenous, and stable. When the obtained GO sheets were dispersed in water for the hydrazine reduction, the brown-colored dispersion of the GO solution became black and the reduced sheets aggregated and eventually precipitated. The reduced sheets precipitated presumably because they became less hydrophilic as a result of the oxygen removal.

The XRD analysis can be used to confirm the graphene preparation. The graphitic structure is usually assigned to a sharp peak at the $2 \theta$ value of $26.5^{\circ}$, which is indexed to the [002] crystal plane [44]. However, when graphite is oxidized to graphite oxide, a new diffraction peak appears at $2 \theta$ of $10.5^{\circ}$, and the diffraction peak disappears [002] [44]. The reduced graphene oxide shows a broad peak that can be fitted using a Lorentzian function into three peaks, which are centered at $2 \theta=20.17^{\circ}, 23,78^{\circ}$ and $25.88^{\circ}$, which correspond to the interlayer distances of $4.47,3.82$ and
$3.53 \AA$, respectively. These XRD results are related to the exfoliation and reduction processes of GO and the processes of removing intercalated water molecules and the oxide groups $[29,45]$. The observed broaden peak indicates the smaller crystalline size of graphene in the single-layer or few-layer structure. Accordingly, from Fig. 6, which represents the XRD pattern of the obtained powder, one can claim that the obtained powder is composed of graphene. Thus, the chemical procedure to prepare graphene was successfully achieved.

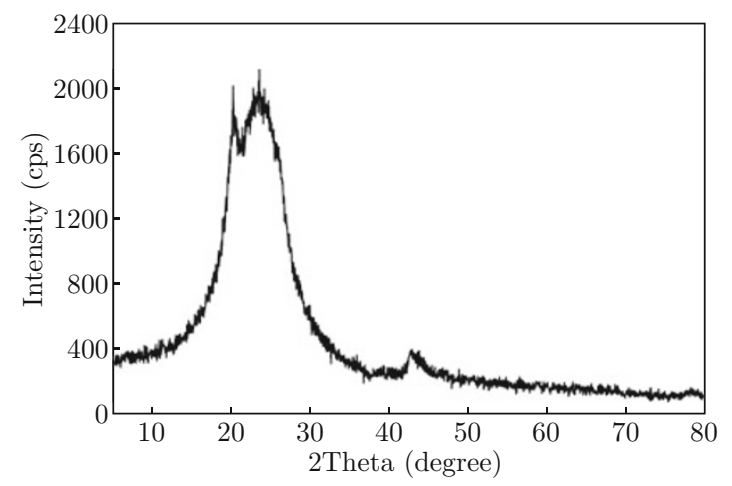

Fig. 6 XRD results for the reduced graphene oxide.

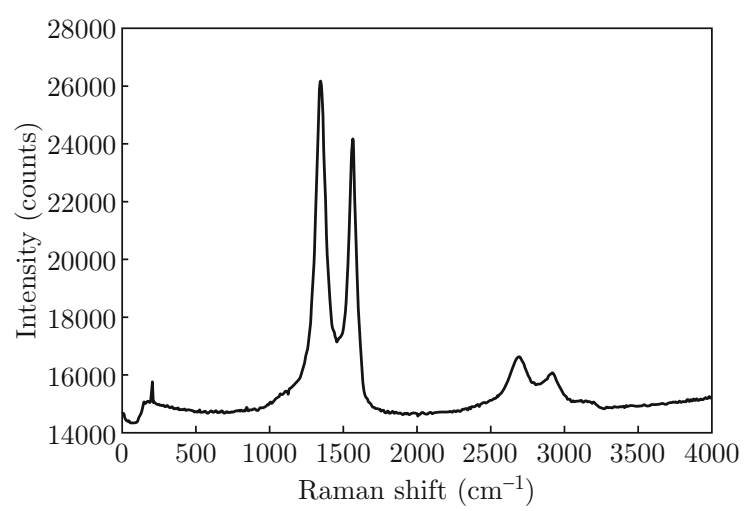

Fig. 7 Raman shift for the prepared reduced graphene oxide.

Raman spectroscopy is a spectroscopic technique that is used to observe the vibrational, rotational, and other low-frequency modes in compounds. Most graphene preparation reports chose Raman spectroscopy to confirm their conclusions. The Raman spectrum of the pristine graphite displays a prominent $G$ peak at $1581 \mathrm{~cm}^{-1}$, which corresponds to the firstorder scattering of the $E_{2 g}$ mode [46]. In the Raman spectrum of GO, the $G$ band is broadened and shifted to $1594 \mathrm{~cm}^{-1}$. In addition, the $D$ band at $1363 \mathrm{~cm}^{-1}$ becomes prominent, which indicates that the in-plane $s p 2$ domains become smaller because of the extensive oxidation. The Raman spectrum of the graphene produced from the GO reduction also contains both $G$ and $D$ bands (at $\sim 1580$ and $\sim 1350 \mathrm{~cm}^{-1}$, respectively); however, this graphene has a higher $D / G$ 
intensity ratio than GO. This change suggests a decrease in the average size of the $s p 2$ domains after the exfoliated GO was reduced [46]. Figure 7 displays the Raman spectrum of the prepared reduced graphene oxide. As shown in the figure, the pattern typically matches the standard graphene Raman spectrum; both $G$ and $D$ bands appear at the reported locations. Moreover, its $D / G$ ratio is higher than one, which further supports the formation of graphene.

Figure 8 shows the normal and the HR TEM images for the prepared reduced graphene oxide. The exfoliation clearly appears in Fig. 8(a). Moreover, the HR TEM image in Fig. 8(b) indicates that the formed graphene is multilayer (approximately 3 layers as shown in the image).
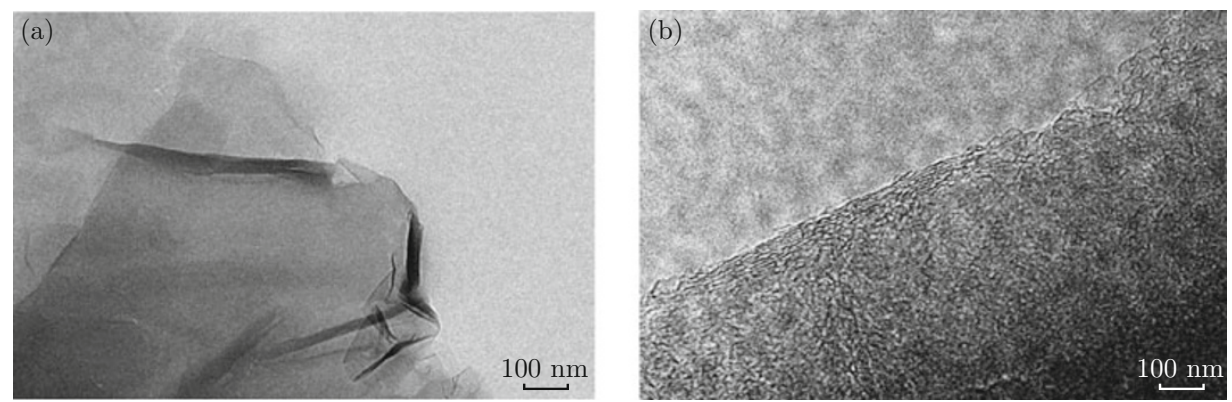

Fig. 8 TEM (a) and HRTEM (b) images for the reduced graphene oxide. The red lines clarify the carbon layers.
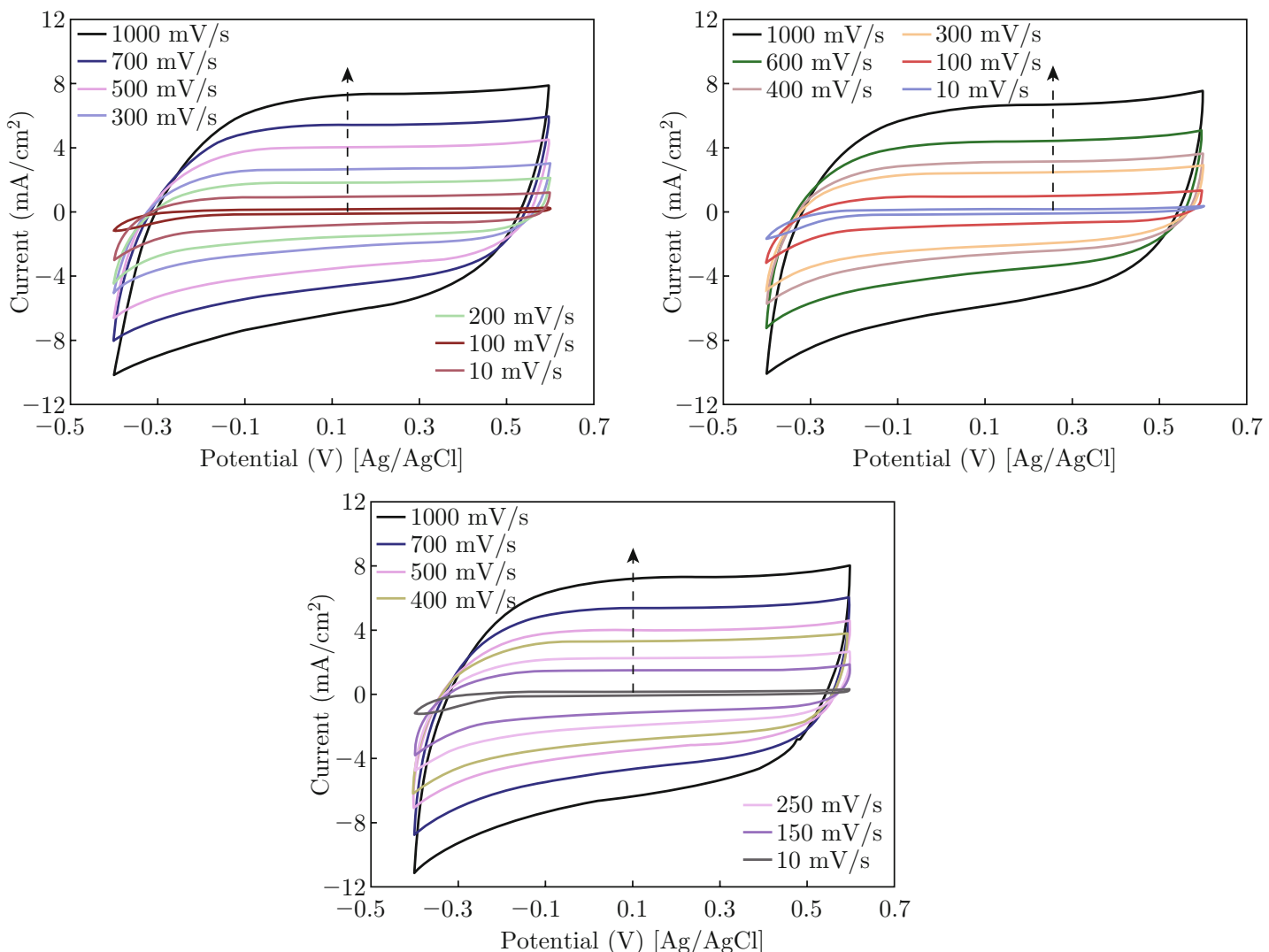

Fig. 9 Cyclic voltammetry results for the prepared $\mathrm{Cd}$-doped $\mathrm{Co} / \mathrm{C}$ electrodes at different scan rates and $\mathrm{NaCl}$ concentration: (a) $0.1 \mathrm{M}$; (b) $0.5 \mathrm{M}$; and (c) $1 \mathrm{M}$.

\section{Capacitive deionization performance}

In general, cyclic voltammetry (CV) is employed to evaluate the potential of the materials used for CDI, and the specific capacitance can be calculated accordingly $[16,47]$. Figure 9 displays the CV results for an electrode (typically, a single electrode is used for all measurements), which was made of the introduced nanoparticles at different $\mathrm{NaCl}$ concentrations $(0.1,0.5$ and $1.0 \mathrm{M}$ ) at selected scan rates from 10 to 1000 $\mathrm{mV} / \mathrm{s}$. Although the introduced NPs have metallic substances, no Faradaic reactions were observed for the used electrodes, which indicates that the ions were adsorbed on the electrode surface by forming an elec- 
tric double layer via a Coulombic interaction instead of electrochemical reactions [15]. The voltammograms are nearly rectangular in the applied potential ranges, where the current quickly leveled off after the potential sweep reversed. An ideal capacitor would create a rectangular shape on a cyclic voltammogram [48]. The shape of the curves becomes more rectangular as the scan rate increases, whereas the voltammetric currents simultaneously increase. These results imply that at low potential scan rates, the electrolytes have sufficient time to accumulate and arrange on the surface, which significantly contributes to the formation of the double-layer capacitance [49]. Therefore, the electrosorption deionization becomes more efficient [47].

Among various carbonaceous materials, graphene attracts the attention of many researchers because of its distinct physical properties. The high surface area and the excellent electrical properties of graphene make it a suitable electrode material in the CDI units. Figure 10 displays the $\mathrm{CV}$ analyses of the introduced $\mathrm{Cd}$-doped $\mathrm{Co} / \mathrm{C} \mathrm{NPs}$ and graphene in the $0.1 \mathrm{M} \mathrm{NaCl}$ solution at different scan rates. As shown in the figures, at low scan rates, graphene exhibits a disordered rectangular shape that may be explained as follows: (i) Faradic reactions occur at these relatively low scan rates [50], (ii) the diffusion of ions in the electrode is limited [51], and
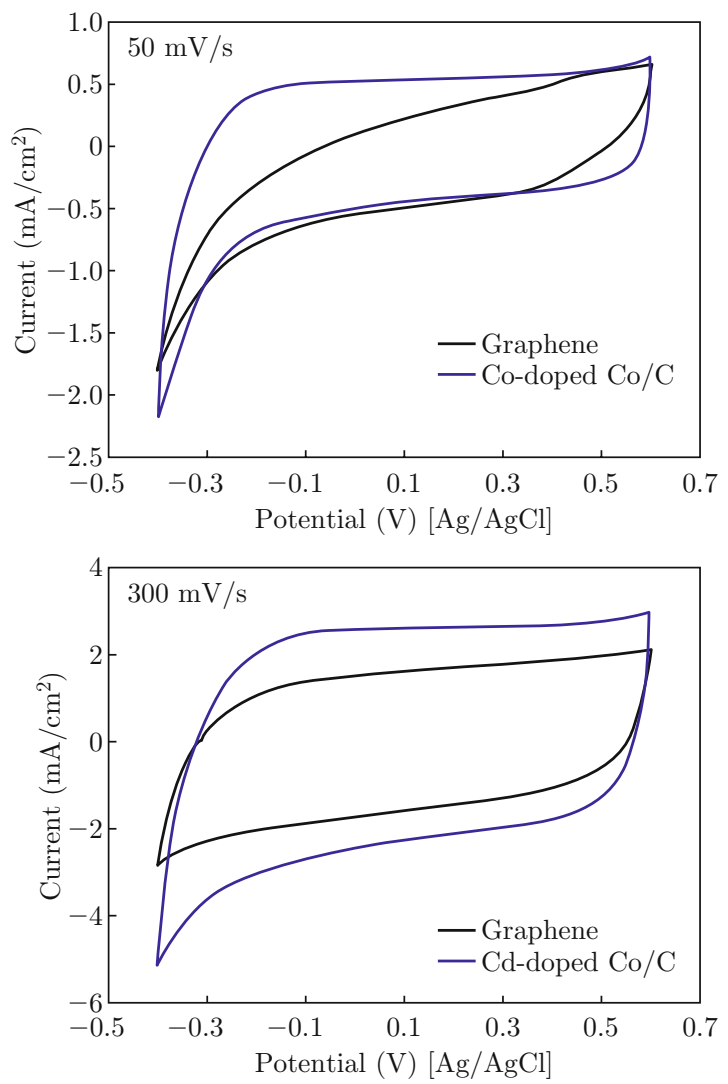

(iii) the reduction of GO to the reduced graphene oxide is incomplete. However, the introduced nanoparticles exhibit a better rectangular shape. Moreover, graphene responses to lower current than the Cd-doped Co/electrode particularly at high scan rates because they have different specific capacitances. Note that activated carbon is the most common material to evaluate the performance of any reported material used in CDI in comparison to the CV results, and graphene and its composites always exhibit higher performances $[3,18,44]$. In our study, activated carbon was also investigated (the CV data are shown), and the performance was small compared to graphene. Thus, we measured the specific capacitances of the introduced encapsulated nanoparticles, the activated carbon and the prepared graphene electrodes at the used scan rates. From the $\mathrm{CV}$, the specific capacitance can be estimated as follows $[17,52]$,

$$
C=\frac{1}{v m} \int \frac{I}{V} d V
$$

where $C$ is the specific capacitance $(\mathrm{F} / \mathrm{g}), v$ is the scan rate $(\mathrm{V} / \mathrm{s}), m$ is the mass of the active material $(\mathrm{g}), I$ is the current density (A), and $V$ is the corresponding voltage $(\mathrm{V})$. In this study, a numerical integration model was established to estimate the specific
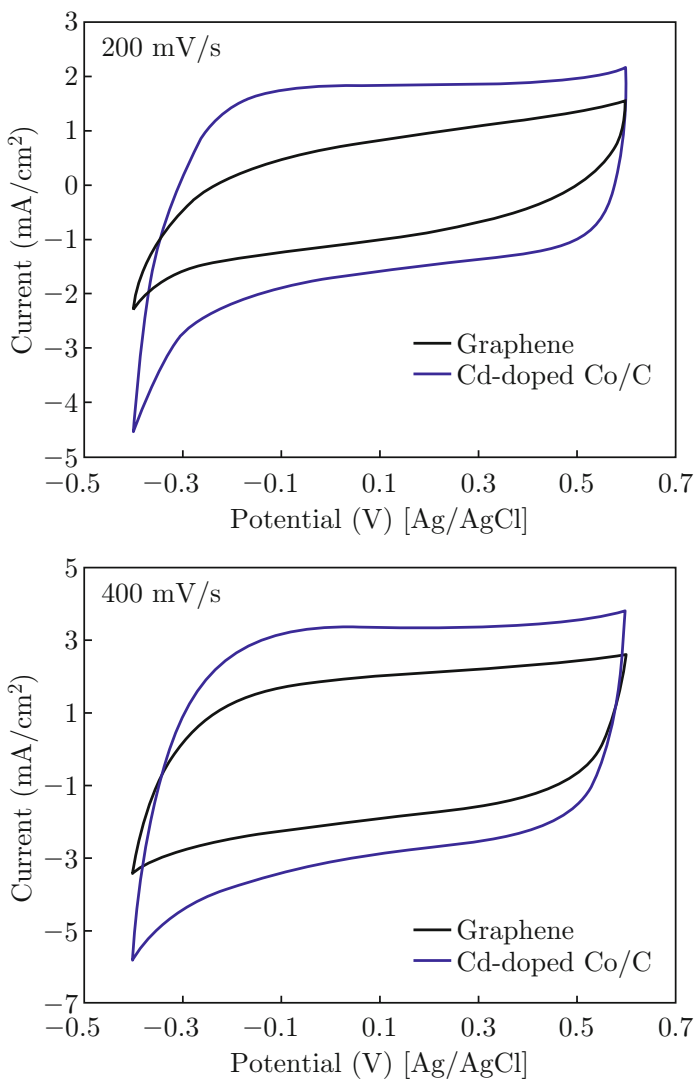

Fig. $10 \mathrm{CV}$ profiles of the introduced Cd-doped Co/C NPs and prepared greaphene electrodes at different scan rates and $\mathrm{NaCl}$ concentration of $0.1 \mathrm{M}$. 
capacitance at every scan rate as follows,

$$
\begin{aligned}
C= & \frac{1}{v m} \sum_{n=1}^{n=N-1}\left[\left(V_{n+1}-V_{n}\right) \times\right. \\
& \left.\left(I_{n+1}+I_{n}\right) /\left(V_{n+1}+V_{n}\right)\right]
\end{aligned}
$$

where $N$ is the number of points in the $\mathrm{CV}$ cycle. As shown in Fig. 11, the introduced NPs have higher corresponding specific capacitance than graphene and activated carbon. At low scan rates, the introduced NPs and graphene have almost identical specific capacitances (206.1 and $205.5 \mathrm{~F} / \mathrm{g}$ at $10 \mathrm{mV} / \mathrm{s}$, respectively), which is higher than the corresponding value of the activated carbon $(120 \mathrm{~F} / \mathrm{g})$. However, at higher scan rates, the introduced NPs have higher specific capacitances than the two carbonaceous materials. In addition, the introduced NPs display good specific capacitance stability at high scan rates, which makes the carbonaceous materials adequate to be used as electrodes in the CDI units. Note that the electrosorption capacitance is generally high at lower scan rates because the diffused ions from the solution can more easily access the electrode surface, which leads to more surface adsorption/desorption of ions [8]. However, at high scan rates, the effective inner-surface adsorption of ions is accordingly reduced.

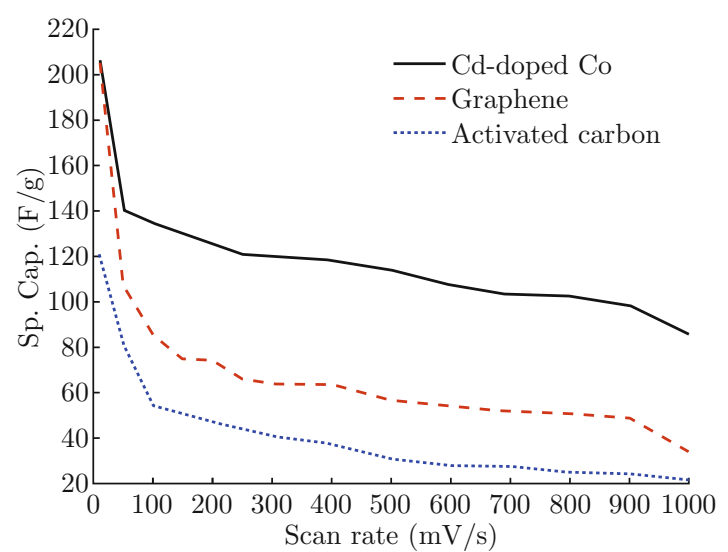

Fig. 11 Specific capacitance for the introduced Cd-doped $\mathrm{Co} / \mathrm{C}$ NPs, the prepared graphene and activated carbon at different scan rates in $0.1 \mathrm{M} \mathrm{NaCl}$ solution.

Overall, one can claim that the successful encapsulation of metallic nanoparticles in a graphite sheath strongly enhances their chemical stability. Furthermore, the metallic counterpart in the introduced NPs improves the specific capacitance compared to the carbonaceous materials. Generally, the enhanced performance of the introduced NPs can be attributed to the high electrical conductivity and the high specific capacitance [26]. Thus, the introduced NPs can be good electrode material in CDI units. In this study, the full electrochemical and physicochemical characterizations are introduced; however, more work is required to properly evaluate the introduced NPs in a prototype scale, which is our plan in the near future.

\section{Conclusion}

Cadmium-doped cobalt nanoparticles in a graphite shell can be prepared by calcination of a powder that is obtained by drying and grinding a sol-gel. The sol-gel is composed of cadmium acetate, cobalt acetate and poly(vinyl alcohol) in an argon atmosphere at $700^{\circ} \mathrm{C}$. Because of the graphite shell, the introduced NPs can be used as effective electrodes in capacitive deionization instruments. Because of the bimetallic counterpart, the introduced NPs perform better than graphene. The metallic constituent enhances the specific capacitance, which positively affects the ion electrosorption process.

\section{Acknowledgement}

This work was financially supported by the National Plan for Science \& Technology (NPST), King Saud University Project No. 11-NAN1460-02. We thank Mr. T. S. Bae and J. C. Lim, KBSI, Jeonju branch, and Mr. Jong- Gyun Kang, Centre for University Research Facility, for taking high-quality FESEM and TEM images, respectively.

\section{References}

[1] A. D. Khawaji, I. K. Kutubkhanah and J. M. Wie, "Advances in seawater desalination technologies", Desalination 221(1), 47-69 (2008). http://dx.doi.org/ $10.1016 / j$.desal . 2007.01.067

[2] T. Welgemoed and C. Schutte, "Capacitive deionization technology ${ }^{\mathrm{TM}}$ : an alternative desalination solution", Desalination 183(1), 327-340 (2005). http:// $\mathrm{dx}$.doi.org/10.1016/j.desal.2005.02.054

[3] H. Li, L. Pan, C. Nie, Y. Liu and Z. Sun, "Reduced graphene oxide and activated carbon composites for capacitive deionization", J. Mater. Chem. 22(31), 15556-15561 (2012). http://dx.doi.org/10. 1039/c2jm32207b

[4] J. K. Edzwald and J. Haarhoff, "Seawater pretreatment for reverse osmosis: chemistry, contaminants, and coagulation", Water Res. 45(17), 54285440 (2011). http://dx.doi.org/10.1016/j.watres. 2011.08 .014

[5] R. Semiat, "Energy issues in desalination processes", Environ.Sci.Technol. 42(22), 8193-8201 (2008). http://dx.doi.org/10.1021/es801330u

[6] A. Subramani, M. Badruzzaman, J. Oppenheimer and J. G. Jacangelo, "Energy minimization strategies and renewable energy utilization for desalination: a review", Water Res. 45(5), 1907-1920 (2011). http:// dx.doi.org/10.1016/j.watres.2010.12.032 
[7] Y. Oren, "Capacitive deionization (CDI) for desalination and water treatment - past, present and future (a review)", Desalination 228(1), 10-29 (2008). http:// dx.doi.org/10.1016/j.desal.2007.08.005

[8] L. Zou, L. Li, H. Song and G. Morris, "Using mesoporous carbon electrodes for brackish water desalination", Water Res. 42(8), 2340-2348 (2008). http://dx. doi.org/10.1016/j.watres.2007.12.022

[9] I. Villar, S. Roldan, V. Ruiz, M. Granda, C. Blanco, R. Menéndez and R. Santamaría, "Capacitive Deionization of $\mathrm{NaCl}$ Solutions with Modified Activated Carbon Electrodes", Energy Fuels. 24(6), 3329-3333 (2010). http://dx.doi.org/10.1021/ef901453q

[10] Y. J. Kim and J. H. Choi, "Enhanced desalination efficiency in capacitive deionization with an ion-selective membrane", Sep. Purif. Technol. 71(1), 70-75 (2010). http://dx.doi.org/10.1016/j.seppur.2009.10.026

[11] L. Zou, G. Morris and D. Qi, "Using activated carbon electrode in electrosorptive deionisation of brackish water", Desalination 225(1), 329-340 (2008). http:// $\mathrm{dx}$.doi.org/10.1016/j.desal.2007.07.014

[12] C. J. Gabelich, T. D. Tran and I. H . Suffet, "Electrosorption of inorganic salts from aqueous solution using carbon aerogels", Environ.Sci.Technol. 36(13), 3010-3019 (2002). http://dx.doi.org/10. 1021/es0112745

[13] P. Xu, J. E. Drewes, D. Heil and G. Wang, "Treatment of brackish produced water using carbon aerogelbased capacitive deionization technology", Water Res. 42(10), 2605-2617 (2008). http://dx.doi.org/10. 1016/j.watres.2008.01.011

[14] L. Pan, X. Wang, Y. Gao, Y. Zhang, Y. Chen and Z. Sun, "Electrosorption of anions with carbon nanotube and nanofibre composite film electrodes", Desalination 244(1), 139-143 (2009). http://dx.doi.org/10.1016/ j.desal.2008.05.019

[15] L. Li, L. Zou, H. Song and G. Morris, "Ordered mesoporous carbons synthesized by a modified sol-gel process for electrosorptive removal of sodium chloride", Carbon 47(3), 775-781 (2009). http://dx.doi.org/ 10.1016/j. carbon. 2008.11.012

[16] H. Li, L. Zou, L. Pan and Z. Sun, "Using graphene nano-flakes as electrodes to remove ferric ions by capacitive deionization", Sep. Purif. Technol. 75(1), 8-14 (2010). http://dx.doi.org/10.1016/j. seppur. 2010.07 .003

[17] J. Yang, L. Zou, H. Song and Z. Hao, "Development of novel $\mathrm{MnO}_{2} /$ nanoporous carbon composite electrodes in capacitive deionization technology", Desalination 276(1), 199-206 (2011). http://dx.doi.org/10.1016/ j.desal.2011.03.044

[18] Z. Wang, L. Yue, Z. T. Liu, Z. H. Liu and Z. Hao, "Functional graphene nanocomposite as an electrode for the capacitive removal of $\mathrm{FeCl} 3$ from water", J. Mater. Chem. 22(28), 14101-14107 (2012). http://dx. doi.org/10.1039/c2jm32175k

[19] D. Zhang, X. Wen, L. Y. Shi, T. Yan and J. Zhang, "Enhanced capacitive deionization of graphene/mesoporous carbon composites", Nanoscale
4(17), 5440-5446(2012). http://dx.doi.org/10. 1039/c2nr31154b

[20] J. Yang, L. Zou and H. Song, "Preparing $\mathrm{MnO}_{2} / \mathrm{PSS} / \mathrm{CNTs}$ composite electrodes by layerby-layer deposition of $\mathrm{MnO}_{2}$ in the membrane capacitive deionisation", Desalination 286, 108-114 (2012). http://dx.doi.org/10.1016/j.desal. 2011. 11.013

[21] M. T. Z. Myint and J. Dutta, "Fabrication of zinc oxide nanorods modified activated carbon cloth electrode for desalination of brackish water using capacitive deionization approach", Desalination 305, 24-30 (2012). http://dx.doi.org/10.1016/j.desal. 2012 . 08.010

[22] M. W. Ryoo and G. Seo, "Improvement in capacitive deionization function of activated carbon cloth by titania modification", Water Res. 37(7), 1527-1534 (2003). http://dx.doi.org/10. 1016/S0043-1354(02) 00531-6

[23] M. W. Ryoo, J. H. Kim and G. Seo, "Role of titania incorporated on activated carbon cloth for capacitive deionization of $\mathrm{NaCl}$ solution", J. Colloid Interface Sci. 264(2), 414-419 (2003). http://dx.doi.org/10.1016/ S0021-9797 (03) 00375-8

[24] N. A. Barakat, K. A. Khalil and H. Y. Kim, "Toward facile synthesizing of diamond nanostructures via nanotechnological approach: Lonsdaleite carbon nanofibers by electrospinning", Mater. Res. Bull. 47(9), 2140-2147 (2012). http://dx.doi.org/ 10.1016/j.materresbull. 2012.06.012

[25] N. A. M. Barakat, B. Kim, S. J. Park, Y. Jo, M. H. Jung and H. Y. Kim, "Cobalt nanofibers encapsulated in a graphite shell by an electrospinning process", J. Mater. Chem. 19(39), 7371-7378 (2009). http://dx. doi.org/10.1039/b904669k

[26] N. A. M. Barakat, A. G. El-Deen, G. Shin, M. Park and H. Y. Kim, "Novel Cd-doped Co/C nanoparticles for electrochemical supercapacitors", Mater. Lett. 99, 168-171 (2013). http://dx.doi.org/10.1016/j. matlet.2013.03.034

[27] S. Park and R. S. Ruoff, "Chemical methods for the production of graphenes", Nat.Nanotechnol. 4(4), 217-224 (2009). http://dx.doi.org/10.1038/nnano. 2009. 58

[28] Y. Xu, H. Bai, G. Lu, C. Li and G. Shi, "Flexible graphene films via the filtration of water-soluble noncovalent functionalized graphene sheets", J. Am. Chem. Soc. 130(18), 5856-5857 (2008). http://dx.doi.org/ 10.1021/ja800745y

[29] W. S. Hummers Jr and R. E. Offeman, "Preparation of graphitic oxide", J. Am. Chem. Soc. 80(6), 1339-1339 (1958). http://dx.doi.org/10.1021/ja01539a017

[30] A. Yousef, N. A. M. Barakat, T. Amna, A. R. Unnithan, S. S. Al-Deyab and H. Yong Kim, "Influence of CdO-doping on the photoluminescence properties of $\mathrm{ZnO}$ nanofibers: effective visible light photocatalyst for waste water treatment", J. Lumin. 132(7), 16681677 (2012). http://dx.doi.org/10.1016/j.jlumin. 2012.02 .031 
[31] N. A. M. Barakat, M. F. Abadir, K. T. Nam, A. M. Hamza, S. S. Al-Deyab, W. I. Baek and H. Y. Kim, "Synthesis and film formation of iron-cobalt nanofibers encapsulated in graphite shell: magnetic, electric and optical properties study", J. Mater. Chem. 21(29), 10957-10964 (2011). http://dx.doi.org/10. 1039/c1jm00052g

[32] N. A. M. Barakat, K. A. Khalil, I. H. Mahmoud, M. A. Kanjwal, F. A. Sheikh and H. Y. Kim, "CoNi Bimetallic Nanofibers by Electrospinning: Nickel-Based Soft Magnetic Material with Improved Magnetic Properties", J. Phys. Chem. C 114(37), 15589-15593 (2010). http://dx.doi.org/10.1021/jp1041074

[33] N. A. M. Barakat, B. Kim and H. Y. Kim, "Production of Smooth and Pure Nickel Metal Nanofibers by the Electrospinning Technique: Nanofibers Possess Splendid Magnetic Properties", J. Phys. Chem. C 113(2), 531-536 (2008). http://dx.doi.org/10.1021/ jp805692r

[34] B. Małecka, "Thermal decomposition of $\mathrm{Cd}$ $\left(\mathrm{CH}_{3} \mathrm{COO}\right)_{2} \cdot \mathrm{H}_{2} \mathrm{O}$ studied by a coupled TG-DTA-MS method", J. Therm. Anal. Calorim. 78(2), 535544 (2004). http://dx.doi.org/10.1023/B: JTAN. $0000046117.25037 .5 a$

[35] T. Wanjun and C. Donghua, "Mechanism of thermal decomposition of cobalt acetate tetrahydrate", Chem. Pap. 61(4), 329-332 (2007). http://dx.doi.org/10. 2478/s11696-007-0042-3.

[36] N. McIntyre and M. Cook, "X-ray photoelectron studies on some oxides and hydroxides of cobalt, nickel, and copper", Anal. Chem. 47(13), 2208-2213 (1975). http://dx.doi.org/10.1021/ac60363a034

[37] G. Hota, S. Idage and K. C. Khilar, "Characterization of nano-sized CdS- $\mathrm{Ag}_{2} \mathrm{~S}$ core-shell nanoparticles using XPS technique", Colloids Surf. A 293(1), 512 (2007). http://dx.doi.org/10.1016/j.colsurfa. 2006.06 .036

[38] A. Gulino, F. Castelli, P. Dapporto, P. Rossi and I. Fragalá, "Synthesis and characterization of thin films of cadmium oxide", Chem. Mater. 14(2), 704-709 (2002). http://dx.doi.org/10.1021/cm011175q

[39] J. Chang, R. S. Mane, D. Ham, W. Lee, B. W. Cho, J. K. Lee and S. H. Han, "Electrochemical capacitive properties of cadmium oxide films", Electrochim. Acta 53(2), 695-699 (2007). http://dx.doi.org/10.1016/ j.electacta.2007.07.056

[40] Pierre Delhaes, "Graphite and Precursors (World of Carbon)", CRC Press, Edition 1 (2001).

[41] S. Stankovich, D. A. Dikin, R. D. Piner, K. A. Kohlhaas, A. Kleinhammes, Y. Jia, Y. Wu, S. B. T. Nguyen and R. S. Ruoff, "Synthesis of graphenebased nanosheets via chemical reduction of exfoliated graphite oxide", Carbon 45(7), 1558-1565 (2007). http://dx.doi.org/10.1016/j.carbon.2007.02.034

[42] Y. Zhu, S. Murali, W. Cai, X. Li, J. W. Suk, J. R. Potts and R. S. Ruoff, "Graphene and graphene oxide: synthesis, properties, and applications", Adv. Mater. 22(35), 3906-3924 (2010). http://dx.doi.org/ 10. 1002 /adma. 201001068

[43] S. Stankovich, R. D. Piner, X. Chen, N. Wu, S. T. Nguyen and R. S. Ruoff, "Stable aqueous dispersions of graphitic nanoplatelets via the reduction of exfoliated graphite oxide in the presence of poly(sodium 4-styrenesulfonate)", J. Mater. Chem. 16(2), 155-158 (2006). http://dx.doi.org/10.1039/b512799h

[44] C. F. Chang, Q. D. Truong and J. R. Chen, "Graphene sheets synthesized by ionic-liquid-assisted electrolysis for application in water purification", Appl. Surf. Sci. 264, 329-334 (2013). http://dx.doi.org/10.1016/j . apsusc. 2012.10.022

[45] A. B. Bourlinos, D. Gournis, D. Petridis, T. Szabó, A. Szeri and I. Dékány, "Graphite oxide: chemical reduction to graphite and surface modification with primary aliphatic amines and amino acids", Langmuir 19(15), 6050-6055 (2003). http://dx.doi.org/ $10.1021 / \mathrm{la} 026525 \mathrm{~h}$

[46] F. Tuinstra and J. Koenig, "Characterization of graphite fiber surfaces with Raman spectroscopy", J. Compos. Mater. 4(4), 492-499 (1970). http://jcm. sagepub. com/content/4/4/492

[47] G. Wang, C. Pan, L. Wang, Q. Dong, C. Yu, Z. Zhao and J. Qiu, "Activated carbon nanofiber webs made by electrospinning for capacitive deionization", Electrochim. Acta 69(1), 65-70(2012). http://dx.doi. org/10.1016/j.electacta.2012.02.066

[48] B. H. Park and J. H. Choi, "Improvement in the capacitance of a carbon electrode prepared using water-soluble polymer binder for a capacitive deionization application", Electrochim. Acta 55(8), 2888-2893 (2010). http://dx.doi.org/10.1016/j. electacta.2009.12.084

[49] J. Chmiola, G. Yushin, R. K. Dash, E. N. Hoffman, J. E. Fischer, M. W. Barsoum and Y. Gogotsi, "Doublelayer capacitance of carbide derived carbons in sulfuric acid", Electrochem. Solid-State Lett. 8(7), A357-A360 (2005). http://dx.doi.org/10.1149/1.1921134

[50] L. L. Zhang, T. Wei, W. Wang and X. Zhao, "Manganese oxide-carbon composite as supercapacitor electrode materials", Micropor. Mesopor. Mater. 123(1), 260-267 (2009). http://dx.doi.org/10.1016/ j.micromeso. 2009.04.008

[51] J. Li, Q. M. Yang and I. Zhitomirsky, "Nickel foambased manganese dioxide-carbon nanotube composite electrodes for electrochemical supercapacitors", J. Power Sources 185(2), 1569-1574 (2008). http://dx. doi.org/10.1016/j.jpowsour. 2008.07.057

[52] A.G.El-Deen, N.A. Barakat, K.A. Khaliland H.Y. Kim, "Development of Multi-Channel Carbon Nanofibers as Effective Electrosorptive Electrodes for a Capacitive Deionization Process", J. Mater. Chem. A 1(36), 11001-11010 (2013). http://dx.doi.org/ 10.1039/C3TA12450A 